\title{
Evaluación de la expresión de marcadores asociados a linfocitos T cooperadores del tipo Th1, Th2, Th17 y Treg en biopsias de pterigión primario
}

\section{Evaluation of the expression of T helper lymphocytes markers associated with Th1, Th2, Th17 and Treg cells in primary pterygium biopsies}

\author{
Carlos Daniel Díaz-Palomera ${ }^{1}$ Ramses Rosales-Díaz', Abril Bernardette Martínez-Rizo, \\ Beatriz Alvarado-Castillo ${ }^{2}$, Arturo Santos ${ }^{3}$ y José Navarro-Partida1* \\ ${ }^{1}$ Unidad Académica de Medicina, Universidad Autónoma de Nayarit, Tepic, Nay.; ${ }^{2} I M S S$, Centro Médico Nacional de Occidente, Guadalajara, Jal.; \\ ${ }^{3}$ Tecnológico de Monterrey, División de Biotecnología y Salud, Campus Guadalajara, Guadalajara, Jal. México
}

\begin{abstract}
Resumen
Introducción: Diversas citocinas proinflamatorias y factores de crecimiento han sido relacionados con la patogenia de pterigión (Pt); sin embargo, el efecto de las citocinas producidas por los linfocitos $T$ cooperadores (Th) ha sido pobremente estudiado. Hasta la fecha, sólo la interleucina (IL) 4 de los linfocitos Th se ha relacionado con la recurrencia del Pt. Por lo tanto, este estudio se llevó a cabo para evaluar la expresión de las citocinas Th y los factores de transcripción Th en Pt primario. Métodos: Se obtuvieron biopsias de Pt de 28 ojos de 28 pacientes mexicanos sometidos a escisión primaria de Pt con autoinjerto conjuntival. Se utilizaron biopsias conjuntivales de 8 pacientes sometidos a cirugía de cataratas como

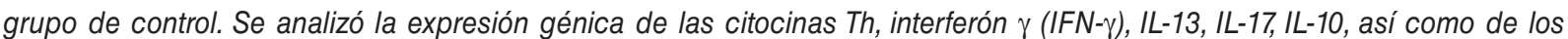
factores de transcripción Th; T-bet, GATA3, Foxp3 y RORyt, mediante retrotranscripción-reacción en cadena de la polimerasa

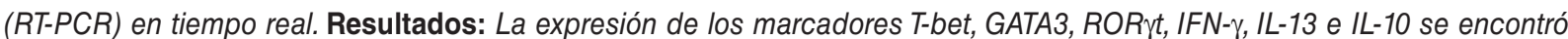
disminuida en Pt. La expresión de IL-17 y Foxp3 se encontró aumentada hasta seis veces en Pt en comparación con la conjuntiva sana $(p<0.0001)$, siendo este incremento dependiente del tamaño del Pt. Conclusiones: El aumento de la expresión de IL-17 y Foxp3 en muestras de Pt sugieren la presencia de linfocitos Th IL-17+Foxp3+, un subconjunto de la población Th con la capacidad de suprimir la proliferación de células $T$ y de promover la progresión tumoral. Por lo tanto, las células Th IL-17+Foxp3 ${ }^{+}$podrían estar implicadas en la aparición y el crecimiento del Pt.
\end{abstract}

Palabras clave: Pterigión primario. Células T cooperadoras. Citocinas. Marcadores de células T.

\section{Abstract}

Introduction: Various pro-inflammatory cytokines and growth factors have been implicated in the pathogenesis of pterygium (Pt); however, the effect of cytokines produced by helper T lymphocytes (Th) has been poorly studied. To date, only interleukin 4 (IL-4) from Th lymphocytes has been linked to Pt recurrence. Therefore, this study aimed to evaluate the expression of Th cytokines and Th transcription factors in primary Pt. Methods: Pt biopsies were obtained from 28 eyes of 28 Mexican patients undergoing primary excision of Pt with conjunctival autograft. Conjunctival biopsies of 8 patients undergoing cataract 
surgery were used as the control group. Gene expression of Th cytokines - interferon gamma (IFN- $)$, IL-13, IL-17, IL-10-, as well as gene expression of Th transcription factors T-bet, GATA3, Foxp3 and RORit, was analyzed by real-time RT-PCR. Results: We found decreased expression of markers T-bet, GATA3, RORYt, IFN- $\gamma$, IL-13 and IL-10 in Pt. We found a sixfold increase in the expression of IL-17 and Foxp3 in Pt compared to healthy conjunctiva $(p<0.0001)$; this increase was dependent on Pt size. Conclusions: The increased expression of IL-17 and Foxp3 in Pt samples suggests the presence of IL-17+ Foxp $3^{+}$Th lymphocytes, a subset of the Th population with the ability to suppress $T$ cell proliferation and promote tumor

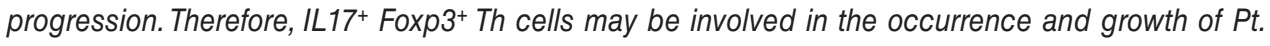

Key words: Primary pterygium. T helper cells. Cytokines. T cells markers.

\section{Introducción}

El Pt es considerado una tumoración epitelial benigna de la córnea cuya característica primordial es la alteración focal del limbo esclerocorneal. Su presencia se asocia a síntomas de malestar ocular, como ardor, irritación, lagrimeo y sensación de cuerpo extraño. La visión suele afectarse en estadios avanzados como resultado del astigmatismo inducido y la obstrucción del eje visual. En raras ocasiones y sólo en casos severos se forma un simbléfaron que limita la movilidad ocular y produce diplopía. Es más prevalente en las zonas ecuatoriales y su aparición está relacionada directamente con la exposición a la radiación ultravioleta, la inflamación y otros factores irritantes. Aunque su etiología no se ha esclarecido, se conoce que la participación del gen p53, la alteración en la apoptosis, la acción de colagenasas y la angiogénesis promueven su desarrollo".

Según la Academia Americana de Oftalmología, el Pt se puede clasificar por su ocurrencia en: a) Pt primario, aquel que va a ser operado por primera vez, y b) Pt recidivante, el que ha recibido uno o más tratamientos quirúrgicos previos, independientemente del método utilizado. De acuerdo con su extensión, el Pt se divide en: a) grado I, cuando llega al limbo esclerocorneal, invadiendo hasta $1 \mathrm{~mm}$; b) grado II, invade la córnea más de $1 \mathrm{~mm}$, sin llegar al borde pupilar; c) grado III, Ilega al borde pupilar, y d) grado IV, sobrepasa el borde pupilar ${ }^{2}$.

Histológicamente, el Pt se define como una hiperplasia fibrovascular de carácter benigno de la conjuntiva bulbar que invade la córnea, con infiltración leucocitaria que incluye a los linfocitos Th CD4+. Los linfocitos Th son capaces de diferenciarse en subpoblaciones distintas, la cuales se caracterizan por el perfil de citocinas que producen y los factores de transcripción que dirigen su diferenciación, que en conjunto pueden denominarse marcadores Th. Los marcadores específicos de los linfocitos Th1 son el IFN- $\gamma$ y el factor de transcripción T-bet; para los Th2, la IL-13 y el factor transcripcional GATA3; para la subpoblación Th17, la IL-17 y el factor ROR $\gamma$ t, y para el linfocito Treg, la IL-10 y el factor Foxp3 ${ }^{3-5}$.

El perfil de citocinas producidas por cada subpoblación de linfocito Th se asocia con diferentes actividades biológicas e incluso con enfermedades específicas. Por ejemplo, las células Th1 son responsables de las respuestas inmunes mediadas por células, mientras que las Th2 son responsables de la inmunidad humoral. Cuando se presenta en algún tejido una respuesta Th1 excesiva, se produce daño tisular intenso; en cambio, la respuesta Th2 excesiva se asocia a atopia e hipersensibilidad ${ }^{3,4}$. En cuanto a las subpoblaciones Th17, se ha descrito su papel en la promoción y acentuación de la respuesta inflamatoria, y ha sido involucrada con fenómenos de autoinmunidad $^{3,4}$. Por último, diversos estudios han identificado a las células Treg como inmunorreguladores en gran número de enfermedades inflamatorias y autoinmunes, incluyendo asma, esclerosis múltiple y diabetes de tipo $1^{6-8}$.

A pesar del conocimiento actual sobre las subpoblaciones de linfocitos Th y su efecto biológico en diferentes enfermedades, el efecto de las citocinas Th en la patogenia del Pt ha sido pobremente analizado. En reportes recientes se sugiere que la expresión de la IL-4 pudiera estar asociada con la recidiva del $\mathrm{Pt}^{9}$.

Aunque de naturaleza benigna, el Pt es considerado un problema de salud pública debido a su alta prevalencia y a los costos derivados de su atención ${ }^{10}$, por lo que se requieren estudios que aporten progresos en el entendimiento de su patogenia deseables debido a que permitirán la identificación de blancos terapéuticos y el diseño de estrategias preventivas y terapéuticas eficaces. Dado que la expresión de citocinas Th y su relación con Pt no han sido dilucidadas, en el presente trabajo revisamos la expresión de marcadores de linfocitos Th en biopsias de Pt primario con el fin de impactar en el conocimiento existente sobre la patogenia de esta entidad clínica. 


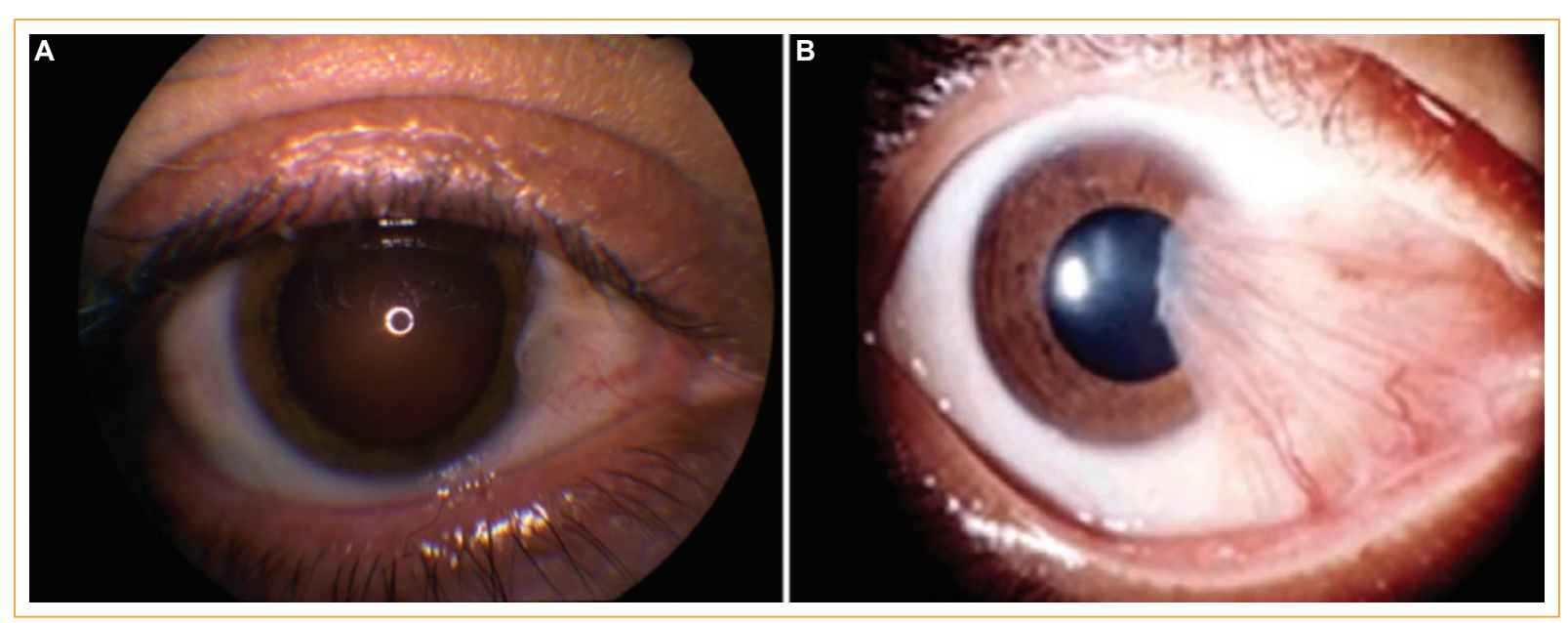

Figura 1. Comparación del grado de extensión de Pt primario. A: Pt grado I. B: Pt grado IV.

\section{Metodología}

\section{Sede del estudio y consideraciones éticas}

El proyecto tuvo como sede el Hospital de Especialidades del Centro Médico Nacional de Occidente del IMSS, y fue autorizado para su ejecución por el Comité Local de Investigación en Salud 1301 de dicho nosocomio, con número de registro R-2012-1301-43, por cumplir con los requerimientos metodológicos de ética y de investigación. Es importante enfatizar que durante el desarrollo del proyecto se atendieron cabalmente los principios éticos postulados en la Declaración de Helsinki.

\section{Recolección de biopsias}

Se incluyeron en el estudio biopsias de conjuntiva sana (grupo de control, $\mathrm{n}=8$ ) obtenidas de sujetos sometidos a cirugía electiva de catarata, y biopsias de Pt primario $(n=28$, siete biopsias por cada grado de extensión del Pt primario -grados I-IV-) de sujetos sometidos a escisión monocular de Pt con colocación de autoinjerto conjuntival (Fig. 1). Antes del evento quirúrgico se obtuvo el consentimiento informado de todos los pacientes después de la explicación extensa sobre el objetivo del estudio y sus alcances. Las biopsias obtenidas en el transoperatorio de los diferentes sujetos fueron sumergidas en solución de ARN-later (AppliedByosistems, CA, EE.UU.) con el fin de asegurar la integridad del ARN mensajero. Tras incubación por $12 \mathrm{~h} \mathrm{a} 4^{\circ} \mathrm{C}$, se procedió al almacenaje de la biopsia a $-80^{\circ} \mathrm{C}$ hasta su utilización.

\section{Extracción de ARN total de cabeza de pterigión}

La extracción de ARN total a partir de biopsia de Pt o conjuntiva se realizó mediante la utilización del reactivo de Trizol (Invitrogen, CA, EE.UU.). Dicho reactivo se define como una solución monofásica de fenol y tiocianato de guanidina, y constituye una mejora del método de extracción de ARN total de un solo paso propuesto por Chomczynski y Sacchi ${ }^{11}$. La extracción del ARN total empezó con la adición de $250 \mu \mathrm{l}$ de Trizol a $200 \mathrm{mg}$ de tejido. Tras una breve incubación en hielo (1 min), se homogeneizó la muestra mediante un homogeneizador automático. Posteriormente se adicionó al homogeneizado $150 \mu \mathrm{l}$ de cloroformo para proceder, tras la incubación por 3 min a temperatura ambiente, a la centrifugación de la muestra a $18,000 \mathrm{rcf} / 20 \mathrm{~min}$ a $4{ }^{\circ} \mathrm{C}$ con el fin de separar la muestra en tres fases: fenólica, intermedia y acuosa. Se colectó la fase acuosa. El ARN se obtuvo a partir de la fase acuosa por precipitación mediante la adición de isopropanol (1:4) e incubación por $24 \mathrm{~h} \mathrm{a}-20^{\circ} \mathrm{C}$. Transcurrido el tiempo de incubación, se centrifugó la muestra a $18,000 \mathrm{rcf} / 15 \mathrm{~min}$ a $4{ }^{\circ} \mathrm{C}$ con el fin de obtener un botón de ARN localizado al fondo del microtubo. Se lavó el botón de ARN por tres ocasiones con etanol al $75 \%$. Se eliminó el etanol mediante decantación y se solubilizó el ARN en agua libre de ARNsas. El ARN obtenido se cuantificó mediante espectrofotometría a una longitud de onda de $260 \mathrm{~nm}$ y se verificó su calidad por medio de la relación 260/280. 
Determinación de la expresión génica por retrotranscripción-reacción en cadena de la polimerasa en tiempo real de marcadores Th

Para el análisis de la expresión de genes y marcadores Th se utilizó el método de RT-PCR en un único paso (Invitrogen, CA, EE.UU.). Se tomó un volumen de $2 \mu \mathrm{l}$ de las muestras de ARN total de los grupos de estudio con una concentración de $8 \mathrm{ng} / \mu \mathrm{l}$, el cual fue sometido a una reacción de RT-PCR en tiempo real utilizando sondas específicas para los genes de las citocinas Th (IFN- $\gamma$, IL-10, IL-13 e IL-17), así como para los genes de los factores de transcripción Th (T-bet, GATA3, Foxp3 y RORyt) (AppliedBiosystems, NJ, EE.UU.). La reacción incluyó: taqMix, DyeRox y 2x Mix de reacción. La expresión génica fue normalizada con la expresión del ARN ribosomal $18 \mathrm{~s}$. Las condiciones de temperatura en la reacción de PCR fueron las siguientes: $30 \mathrm{~min}$ a $48^{\circ} \mathrm{C}, 10 \mathrm{~min}$ a $95^{\circ} \mathrm{C}$ y 40 ciclos de $15 \mathrm{~s}$ a $95^{\circ} \mathrm{C}$, alternados con $1 \mathrm{~min}$ a $60{ }^{\circ} \mathrm{C}$. La reacción de PCR y la detección de los productos de amplificación fue llevada a cabo en el termociclador OneStep Plus (AppliedBiosystems, NJ, EE.UU.). La cuantificación relativa de la expresión génica se realizó mediante el método de $2^{-\Delta \Delta C T}$ utilizando al grupo de control como calibrador interno $0^{12,13}$.

\section{Análisis estadístico}

Los datos con distribución no normal fueron analizados mediante las pruebas no paramétricas de Kruskal-Wallis, Dunn's y U de Mann-Whitney. Los datos con distribución normal fueron analizados mediante oneway ANOVA. Las pruebas post-hock, Holm-Sidak y Dunnett se utilizaron para determinar la significancia estadística. Los resultados se presentan como media \pm error estándar. La significancia estadística se definió como un valor de $p<0.05$.

\section{Resultados}

Con el objetivo de determinar la influencia de las subpoblaciones de linfocitos Th en Pt primario, se determinó la expresión génica de diversos marcadores específicos de los linfocitos Th1 (IFN- $\gamma$ y T-bet), Th2 (IL-13 y GATA3), Th17 (IL-17 y ROR $\gamma$ ) y Treg (IL-10 y Foxp3) en biopsias de Pt obtenidas de pacientes con diversos grados de extensión de la tumoración.

Como puede observarse en la figura 2, la expresión de citocinas Th mostró un patrón diferente para

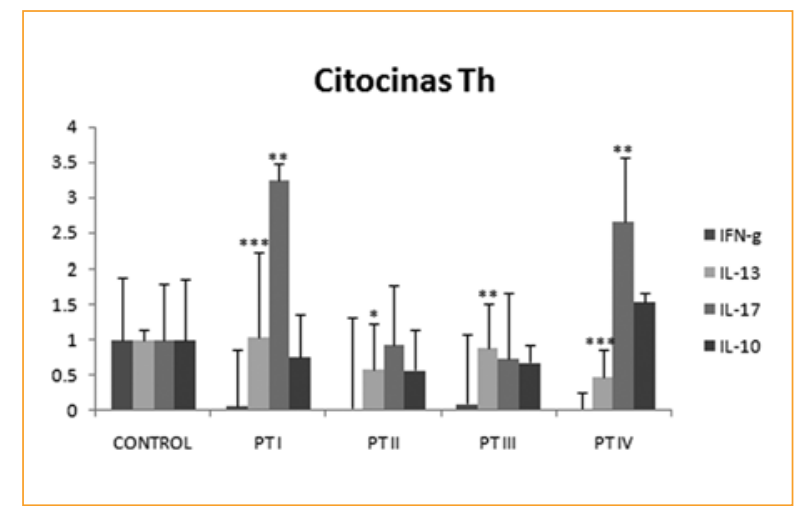

Figura 2. Expresión de citocinas Th. Se observa la expresión génica relativa de las citocinas Th expresada en veces de cambio $\left(2^{-\Delta \Delta C T}\right)$ con relación al control (conjuntiva sana). Los valores presentados corresponden a la media \pm el error estándar. ${ }^{*} \mathrm{p}<0.05 ;{ }^{* *} \mathrm{p}<0.01 ;{ }^{* * *} \mathrm{p}<0.001$.

cada subpoblación, en especial para las subpoblaciones Th2 y Th17. La citocina Th2 IL-13 mostró una disminución significativa en relación con el grupo de control que, además, fue dependiente del grado de extensión; es decir, a mayor grado de extensión, se observó una menor expresión. En lo relativo a la expresión de IL-17 de la subpoblación Th17, la expresión se encontró incrementada con respecto a control en particular en las biopsias de $\mathrm{Pt}$ de los grados I y IV. La expresión de IFN- $\gamma$ e IL-10 (citocinas Th1 y Treg, respectivamente) no presentó diferencias significativas entre los diferentes grupos de estudio.

En relación con la expresión de los factores de transcripción Th (Fig. 3), se encontró un incremento en la expresión de los factores T-bet y Foxp3, el cual fue en declive conforme incrementaba el grado de extensión del Pt. El factor Th2 GATA3 se encontró significativamente disminuido en un patrón que concuerda con la expresión de la citocina Th2 IL-13. Por último, la expresión de RORyt no mostró diferencia significativa entre los grupos.

Es conveniente subrayar que, a diferencia de los marcadores Th2, en los que se encuentra concordancia entre los niveles de su citocina (IL-13) y su factor de transcripción (GATA3), el patrón de expresión del resto de marcadores Th no fue coincidente. Curiosamente, se observó una sobreexpresión de IL-17 (marcador Th17) y Foxp3 (marcador de células Treg) en biopsias de Pt en comparación con las biopsias de conjuntiva sana $(p<0.0001)$. 


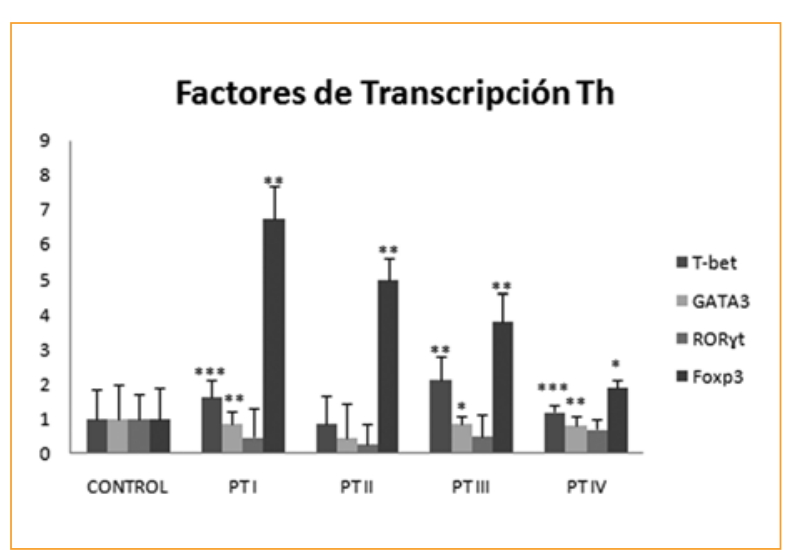

Figura 3. Expresión de factores de transcripción Th. Se observa la expresión génica relativa de los factores de transcripción Th expresada en veces de cambio ( $\left.2^{-\Delta \Delta C T}\right)$ con relación al control (conjuntiva sana). Los valores presentados corresponden a la media \pm el error estándar.

${ }^{*} \mathrm{p}<0.05 ;{ }^{* *} \mathrm{p}<0.01 ;{ }^{* *} \mathrm{p}<0.001$.

\section{Discusión}

Hasta el momento, el conocimiento sobre la expresión de citocinas Th en Pt es limitada, motivo por el cual se decidió realizar la evaluación de la expresión de los marcadores Th en biopsias de Pt primario. Como se mostró en los resultados, la expresión de los genes de T-bet, GATA3, ROR $\gamma$ t, IFN- $\gamma$, IL-13 e IL-10 se encontró disminuida en $\mathrm{Pt}$, mientras que la expresión de IL-17 y Foxp3 se encontró aumentada hasta seis veces en las biopsias de Pt en comparación con las conjuntivas sanas analizadas $(p<0.0001)$, siendo este incremento dependiente del grado de extensión del Pt (a mayor grado de extensión, menor expresión).

En el grado I de extensión de Pt se puede observar que la expresión de IL-13 se encuentra al mismo nivel que en las conjuntivas sanas. Algunos estudios sugieren que IL-13 es la citocina Th2 predominante en la superficie ocular normal, razón por la cual encontramos una expresión basal en los tejidos que puede modificarse al aumentar el grado de extensión de $\mathrm{Pt}^{14}$. Por otro lado, se ha descrito en biopsias de Pt obtenidas de pacientes atópicos y no atópicos un aumento significativo en la expresión de IL-4 y una disminución evidente de IFN- $\gamma$, lo que concuerda con los resultados observados en nuestras muestras ${ }^{15}$. Es importante enfatizar que, a pesar de que la expresión de IFN- $\gamma$ se encuentra muy disminuida en todos los grados de extensión del Pt, podemos observar que, en las biopsias correspondientes a los grados I, III y IV, la expresión del factor transcripcional Th1 (T-bet) se encuentra aumentada, lo que sugiere que en el microambiente del Pt existe el estímulo principal de la diferenciación Th1 (IL-12), pero no en la cantidad suficiente para inducir la polarización ${ }^{3,16}$. En contraste, la expresión del factor transcripcional Th2 (GATA3) se encuentra disminuida en las mismas biopsias de $\mathrm{Pt}$, lo que puede deberse al efecto inhibitorio de T-bet sobre la expresión de GATA3 ${ }^{16-19}$. El aumento de la expresión de IL-17 y Foxp3 en muestras de Pt sugiere la presencia de linfocitos Th IL-17+Foxp3 ${ }^{+}$, un subconjunto de la población Th recientemente descrito con la capacidad de suprimir la proliferación de células $\mathrm{T}$ y de promover la progresión tumora ${ }^{20}$. En estudios realizados previamente en pacientes con Pt primario y recidivante, se encontró que ambos mostraban un nivel elevado de IL-17 en la película lagrimal, por lo que se sugiere que podría estar asociado con su causa y recurrencia ${ }^{21}$.

Las células Th IL-17+Foxp3+ han sido identificadas en diversas enfermedades humanas, como la enfermedad inflamatoria intestinal ${ }^{22}$, tumores de colon ${ }^{23}$, periodontitis ${ }^{24}$, psoriasis ${ }^{25}$ yartritis reumatoide ${ }^{26}$. Característicamente estas células conservan la actividad inmunosupresora de los linfocitos Treg, al tiempo que adquieren capacidad efectora por la producción de IL-1720. Es conocido el hecho de que el microambiente en el que se encuentren los linfocitos Th precursores (naive) determina el fenotipo del linfocito Th diferenciado; sin embargo, es importante también considerar que los linfocitos Th efectores pueden modificar su fenotipo en respuesta a los estímulos circundantes. Los linfocitos Th IL-17+Foxp3 ${ }^{+}$aparecen en respuesta al estímulo de IL-1//IL-6/IL-23 sobre los linfocitos Treg ${ }^{27,28}$. Podemos observar que en los grados de extensión II y III la expresión de IL-17 se encuentra disminuida, lo que podría explicarse por el fenómeno anteriormente descrito, es decir, en estos grados de extensión del Pt el microambiente no es el ideal para la diferenciación de células IL-17+Foxp3+

En nuestro estudio observamos la inhibición de citocinas Th1 y Th2 (en particular de IL-13), lo que es consistente con la actividad supresora supuesta de los linfocitos Th IL-17+Foxp3 ${ }^{+}$, al mismo tiempo que documentamos que la expresión de estos marcadores se asoció con la progresión del Pt, lo que coincide con la actividad tumorigénica de esta población celular; sin embargo, es necesario enfatizar que, si bien en este proyecto logramos identificar la sobreexpresión de IL17 y Foxp3 en biopsias de Pt, es necesario realizar estudios complementarios con el fin de corroborar la presencia de esta subpoblación celular, por ejemplo, mediante la identificación de marcadores de superficie específicos en células obtenidas de los tejidos. En 
conclusión, los linfocitos Th IL17+Foxp3 ${ }^{+}$podrían estar implicados en el desarrollo y la progresión del Pt; sin embargo, se requieren estudios complementarios con el fin de ratificar y ampliar estos hallazgos.

\section{Responsabilidades éticas}

Protección de personas y animales. Los autores declaran que los procedimientos seguidos se conformaron a las normas éticas del comité de experimentación humana responsable y de acuerdo con la Asociación Médica Mundial y la Declaración de Helsinki.

Confidencialidad de los datos. Los autores declaran que han seguido los protocolos de su centro de trabajo sobre la publicación de datos de pacientes.

Derecho a la privacidad y consentimiento informado. Los autores han obtenido el consentimiento informado de los pacientes y/o sujetos referidos en el artículo. Este documento obra en poder del autor de correspondencia.

\section{Bibliografía}

1. Detorakis ET, Spandidos DA. Pathogenetic mechanisms and treatment options for ophthalmic pterygium: trends and perspectives (Review). Int J Mol Med. 2009;23(4):439-47

2. Johnston SC, Williams PB, Sheppard JDJ. A Comprehensive System fo Pterygium Classification. Investigative Ophthalmology \& Visual Science. 2004;45:2940.

3. Raphael I, Nalawade S, Eagar TN, Forsthuber TG. T cell subsets and their signature cytokines in autoimmune and inflammatory diseases. Cytokine. 2015;74(1):5-17.

4. Hirahara K, Nakayama T. CD4+ T-cell subsets in inflammatory diseases: beyond the Th1/Th2 paradigm. Int Immunol. 2016;28(4):163-71.

5. Kumagai J, Hirahara K, Nakayama T. Pathogenic Th cell subsets in chronic inflammatory diseases. Nihon Rinsho Meneki Gakkai Kaishi. 2016;39(2):114-23.

6. Robinson DS. Regulatory T cells and asthma. Clin Exp Allergy. 2009; 39(9):1314-23.

7. Jaeckel E, Mpofu N, Saal N, Manns MP. Role of regulatory T cells for the treatment of type 1 diabetes mellitus. Horm Metab Res. 2008;40(2):126-36.
8. Costantino CM, Baecher-Allan C, Hafler DA. Multiple sclerosis and regulatory T cells. J Clin Immunol. 2008;28(6):697-706.

9. Mackenzie FD, Hirst LW, Battistutta D, Green A. Risk analysis in the development of pterygia. Ophthalmology. 1992:99(7):1056-61.

10. Harrison M. Cost of pterygium. Clin Exp Ophthalmol. 2002;30(4):312.

11. Chomczynski P, Sacchi N. Single-step method of RNA isolation by acid guanidinium thiocyanate-phenol-chloroform extraction. Anal Biochem. 1987:162(1):156-9.

12. Livak KJ, Schmittgen TD. Analysis of relative gene expression data using real-time quantitative PCR and the 2(-Delta Delta $\mathrm{C}(\mathrm{T})$ ) Method. Methods. 2001;25(4):402-8

13. Yuan JS, Reed A, Chen F, Stewart CN Jr. Statistical analysis of real-time PCR data. BMC Bioinformatics. 2006;7:85.

14. Pflugfelder SC, Corrales RM, de Paiva CS. T helper cytokines in dry eye disease. Exp Eye Res. 2013:117:118-25.

15. Gharaee $\mathrm{H}$, Shaayegan MR, Khakzad MR, et al. The expression of vascular endothelial growth factor in pterygium tissue of atopic patients. Int Ophthalmol. 2014;34(6):1175-81.

16. Usui T, Preiss JC, Kanno Y, et al. T-bet regulates Th1 responses through essential effects on GATA-3 function rather than on IFNG gene acetylation and transcription. J Exp Med. 2006;203(3):755-66.

17. Yates A, Callard R, Stark J. Combining cytokine signalling with T-bet and GATA-3 regulation in Th1 and Th2 differentiation: a model for cellular decision-making. J Theor Biol. 2004;231(2):181-96.

18. Szabo SJ, Kim ST, Costa GL, et al. A novel transcription factor, T-bet, directs Th1 lineage commitment. Cell. 2000;100(6):655-69.

19. Hwang ES, Szabo SJ, Schwartzberg PL, Glimcher LH. T helper cell fate specified by kinase-mediated interaction of T-bet with GATA-3. Science. 2005;307(5708):430-3

20. Du R, Zhao H, Yan F, Li H. IL-17+Foxp3+ T cells: an intermediate differentiation stage between Th17 cells and regulatory T cells. J Leukoc Biol. 2014:96(1):39-48.

21. Huang $\mathrm{Y}, \mathrm{He} H$, Sheha $H$, Tseng SC. Ocular demodicosis as a risk factor of pterygium recurrence. Ophthalmology. 2013;120(7):1341-7.

22. Hovhannisyan Z, Treatman J, Littman DR, Mayer L. Characterization of interleukin-17-producing regulatory $T$ cells in inflamed intestinal mucosa from patients with inflammatory bowel diseases. Gastroenterology. 2011;140(3):957-65

23. Ma C, Dong X. Colorectal cancer-derived Foxp3(+) IL-17(+) T cells suppress tumour-specific CD8+ T cells. Scand J Immunol. 2011;74(1):47-51.

24. Okui $T$, Aoki $Y$, Ito $H$, Honda $T$, Yamazaki $K$. The presence of IL-17+/FOXP3+ double-positive cells in periodontitis. J Dent Res. 2012;91(6):574-9.

25. Bovenschen HJ, van de Kerkhof PC, van Erp PE, Woestenenk R, Joosten I, Koenen HJ. Foxp3+ regulatory $T$ cells of psoriasis patients easily differentiate into IL-17A-producing cells and are found in lesional skin. J Invest Dermatol. 2011;131(9):1853-60.

26. Komatsu N, Okamoto K, Sawa S, et al. Pathogenic conversion of Foxp3+ T cells into TH17 cells in autoimmune arthritis. Nat Med. 2014;20(1):62-8.

27. Ueno A, Jijon $\mathrm{H}$, Chan $\mathrm{R}$, et al. Increased prevalence of circulating novel IL-17 secreting Foxp3 expressing CD4+ T cells and defective suppressive function of circulating Foxp3+ regulatory cells support plasticity between Th17 and regulatory T cells in inflammatory bowel disease patients. Inflamm Bowel Dis. 2013;19(12):2522-34.

28. Malmhall C, Bossios A, Radinger M, et al. Immunophenotyping of circulating $T$ helper cells argues for multiple functions and plasticity of $T$ cells in vivo in humans--possible role in asthma. PLoS One. 2012;7(6):e40012. 